\title{
Ärztliche Praxis und wissenschaftlicher Status der Medizin
}

\author{
Peter F. Matthiessen \\ Lehrstuhl für Medizintheorie und Komplementärmedizin, Universität Witten/Herdecke, Deutschland
}

In ihrem Editorial vom Januar 2006 haben H. Walach und A. Michalsen [1] den neuen Untertitel und damit die zukünftige thematische Schwerpunktsetzung der Forschenden KoMPLEMENTÄRMEDIZIN vorgestellt und begründet. Im Zuge des Anliegens eines verstärkten Praxisbezugs würdigen sie die Rolle des Einzelfalls für die Erkenntnisgewinnung in der Medizin und ermuntern Praktiker aller Richtungen, lehrreiche und aussagekräftige Fallberichte einzureichen. Denn, so die Autoren: «In jedem Fall beginnt und endet die wissenschaftliche Bemühung um Erkenntnis in der Praxis bzw. am Einzelfall». Diesen Faden möchte ich aufgreifen und die Frage nach der Rolle der Wissenschaft in der Medizin und nach dem wechselseitigen Verhältnis von Wissenschaft und Praxis weiter verfolgen; denn daraus leiten sich weit reichende Folgen für den Stellenwert der Praxis und für das professionelle Selbstverständnis von Arzt und Therapeut ab.

Im Gegensatz zur Freiheit in der Wahl der Problemstellung als einem zentralen Element der Wissenschaftsfreiheit entspringt ärztliches Erkennen und Handeln keiner frei gewählten Aufgabenstellung, sondern der Konfrontation mit einem von aussen vorgegebenen Problem, das es im Hier und Jetzt zu lösen gilt. So unverzichtbar Wissenschaft und Forschung für die Weiterentwicklung der Medizin und der diagnostischen und therapeutischen Möglichkeiten des Arztes auch sind, konstitutiv für die Medizin ist ein explizit oder implizit geäusserter Behandlungsauftrag des Patienten; Ausgangspunkt ist die einer zwischenmenschlichen Begegnung entstammende Erfahrung einer Notsituation und die daraus resultierende Selbstverpflichtung zur bestmöglichen Hilfestellung. Hierzu braucht der Arzt die Ergebnisse regelwissenschaftlicher Forschung wie die Luft zum Atmen. Aber Wissenschaft ist für ihn nie Selbstzweck, sondern legitimiert sich in dem Masse, als sie bei der Betreuung seiner individuellen Patienten von Wert ist. Das macht deutlich, dass sich das Verhältnis von Wissenschaft und Praxis in der Auffassung der
Medizin als einer angewandten Wissenschaft nicht kongruent abgebildet findet. Denn insoweit sich die Medizin als angewandte Wissenschaft versteht, fokussiert sich ihr Interesse auf die Gewinnung und Berücksichtigung allgemeiner Gesetzmässigkeiten und Regeln. Was sie anstrebt, ist ein vom Erkenntnissubjekt emanzipiertes formales Wissen vom Typus eines «Know what», mit dem idealerweise wertneutrale kausale Erklärungen von Ereigniszusammenhängen gewonnen werden. Ihr Mehrwert für die Praxis ist die Bereitstellung von möglichst gut gesichertem Regelwissen. Kriterien für dessen Anwendung im konkreten Fall lassen sich jedoch nicht innerhalb dieses Ansatzes selbst finden. Denn, und das wusste schon Kant: «Es gibt keine Regeln, wie man Regeln richtig anwendet.» Was also ist die Rationalitätsgrundlage der ärztlichen Praxis? Auf welcher Basis erfolgt die Transformation theoretischen und überwiegend probabilistischen Wissens in die Faktizität ärztlichen Handelns am unaustauschbaren Fall in einer nicht wiederholbaren Situation? Diese Frage findet sich aber in der Definition und im Selbstverständnis der Medizin als angewandte Wissenschaft in naiver Selbstverständlichkeit übergangen und so einem unreflektierten Pragmatismus überlassen - mit der Gefahr, dass sich der Erwerb praktischer Kompetenz in der Übernahme von Handlungsgewohnheiten und der Aneignung von Berufstricks erschöpft.

In einer gerade auch für Praktiker höchst lesenswerten medizintheoretischen Untersuchung zum Diagnosebegriff zeigt Wolfgang Wieland [2] auf, dass der Medizin nicht der Status einer angewandten, sondern derjenige einer praktischen Wissenschaft zukommt, die als solche gefordert ist, die Begründung und Rechtfertigung ihres Handelns innerhalb ihrer selbst zu leisten. Den Ergebnissen regelwissenschaftlicher Forschung kommt für die Praxis stets nur Werkzeugcharakter, eine wiewohl unverzichtbare Hilfsfunktion zu. Das gilt auch für die Elaboration einer jeden Krankheitslehre und für die Erstellung einer Diagnose. Sie sind nicht Selbstzweck, son-

\begin{tabular}{ll}
\hline KARGER & @ 2006 S. Karger GmbH, Freiburg \\
Fax +497614520714 & Accessible online at: \\
$\begin{array}{l}\text { E-mail Information@Karger.de } \\
\text { www.karger.com }\end{array}$ & www.karger.com/fok \\
&
\end{tabular}

Prof. Dr. med. Peter F. Matthiessen

Lehrstuhl für Medizintheorie und Komplementärmedizin

Universität Witten/Herdecke

Gerhard-Kienle-Weg 4, 58313 Herdecke, Deutschland

Tel. +49 2330623890

E-mail peter.matthiessen@uni-wh.de 
dern dienen einer rationalen Therapieentscheidung einschliesslich der begründeten Unterlassung einer Therapie. Indem die Diagnose dem Arzt eine verantwortbare Handlungsentscheidung im Hinblick auf einen konkreten Patienten ermöglichen soll, erweist sie sich - entgegen einem verbreiteten Missverständnis - nicht als Ergebnis einer subsumptionslogischen Zuordnung des am einzelnen Kranken Aufgefundenen zu allgemeinen Krankheitskonstrukten, sondern umgekehrt als eine Leistung der begründeten und sachgerechten Anverwandlung allgemeiner Krankheitsbegriffe bzw. allgemeinen Wissens an die individuelle Krankheitssituation eines konkreten Patienten. «Der Einzelfall ist hier um seiner selbst willen von Interesse» [2]. Das ist er aber nur dort, wo es uns gelingt, die Situation des Patienten nicht als defizitäre Abweichung einer allgemeinen Norm aufzufassen, sondern ihn in seiner Besonderheit positiv zu begreifen, also eine Anschmiegung unseres allgemeinen und keineswegs nur nosologischen Wissens an seine Krankheitssituation vorzunehmen. Für die Praxis stellt sich also die Aufgabe der Ent-allgemeinerung und der Re-individualisierung von Regelwissen, die Herausforderung zur Ausbildung der Fähigkeit, auch das unaustauschbar Besondere einer Krankheitssituation gesamthaft zu erfassen. Diese Fähigkeit gründet aber nicht auf formalem Kalkül, sondern entspringt dem Wissenstyp des «Know how», einem dispositionellen, an die erkennende und handelnde Person gebundenen, jedoch durchaus interpersonal kommunizierbaren Wissen und Können, über das der Arzt in dem Masse verfügt, als er sich an der Praxis für die Praxis die entsprechenden Erkenntnisorgane erworben hat [3]. Für Arzt wie Patienten ist eine Diagnose erst dann von Wert, wenn sie geeignet ist zur Beantwortung der Frage: Was ist zu tun? Für den Praktiker stellt die Erstellung einer Diagnose - bedacht oder nicht bedacht - stets eine therapeutische Handlungsentscheidung dar, liegt einer Therapieentscheidung - bemerkt oder unbemerkt stets ein diagnostisches Urteil zugrunde. Im Gegensatz zu einem dekontextualisierenden Vorgehen, nämlich aus dem Gesamt einer Krankheitssituation einzelne Merkmale bzw. nosologische Entitäten herauszuschälen, wie es im Rahmen nomothetischer, auf Verallgemeinerbarkeit zielender Forschung ebenso üblich wie Erkenntnisgewinn bringend ist, finden wir uns in der Praxis herausgefordert, eine kontextualisierende, auch die lebensweltlichen Bedingungen von Krankheit und Kranksein berücksichtigende Diagnostik zu verfolgen. Denn indem der Diagnose die Aufgabe zukommt, als rationalitätsstiftendes Werkzeug für eine individuelle Therapieentscheidung zu dienen, erweist sie sich als Ergebnis einer Aufmerksamkeitsausrichtung, die weit über den Bereich streng nosologiebezogener Beurteilungskriterien hinausreicht. Es zeigt sich, dass so genannte unspezifische Kontextfaktoren, etwa dispositonelle und konstitutionelle Aspekte, die psychosoziale Situation, die prämorbide Persönlichkeitsstruktur, persönliche Präferenzen und Werte, die aktuelle Lebensphase, aber auch die Frage nach der Bedeutung der bisherigen Lebensgeschichte für den Verlauf und den Umgang mit einer
Krankheit und nicht zuletzt die Frage nach der potentiellen Bedeutung der Erkrankung für die weitere Biographie eines Menschen für die Therapiewahl oft von hoher, nicht selten von ausschlaggebender Relevanz sind. Auch daran verdeutlicht sich, dass sich das Diagnostizieren nicht in der Anheftung eines nosologischen Etiketts erschöpft und dass eine individuelle Therapie eine Individualisierung der Diagnose voraussetzt. Dass sich eine dergestalt personotrope Diagnostik neben objektivierenden Akten der Krankheitsregistrierung zugleich auch als Ergebnis eines subtilen zwischenmenschlichen, Empathie-gestützten Gewahrwerdens des - eher zuständlich als gegenständlich erlebten - Krankseins des Patienten erweist, dürfte im Kontext einer ihrem Namen gerecht werden wollenden Humanmedizin kaum rechtfertigungspflichtig sein. Das ist aber keineswegs ein Plädoyer dafür, den Kranken nur in seiner Singularität in den Blick zu nehmen. Gerade das Vorgehen, den Krankheitsprozess eines Patienten nicht nur als Fall für sich, sondern als Fall einer allgemein-nosologischen Gesetz- bzw. Regelhaftigkeit zu begreifen, macht einen wesentlichen Teil der Professionalität des Arztes aus. Und gerade darauf beruht ja das - freilich stets mit Vorsicht zu verfolgende - Generalisierungspotential jeder guten Kasuistik.

Das Falldenken des Praktikers ist ein Denken in Gestaltzusammenhängen, ein «ratiomorphes» [4] Erfassen von Typologien, auf die er sich auch dort bezieht, wo er vom Atypischen und Individuellen spricht. Der Einzelfall charakterisiert sich so durch seine Janusgesichtigkeit: in der Herausarbeitung von Typologien wird er zum potentiellen Sprungbrett für generalisierende Forschung, zugleich ist er aber auch Ausgangspunkt für ein idiographisches Vorgehen, das - über die (biologische und psychologische) Eigengesetzlichkeit von Krankheitsverläufen hinaus - nach der geschichtlichen Dimension des Menschen in Gesundheit und Krankheit fragt, worunter sich der Blick auf die Krankheits«geschichte» um denjenigen auf die Kranken- und darüber hinaus auf die Lebensgeschichte eines Menschen erweitert. Dies scheint mir insbesondere dort unverzichtbar, wo es darum geht, im Zuge der Wiederentdeckung des Phänomens «Salutogenese» [5] ein einseitig pathogenetisch orientiertes Denken um einen diagnostischen Blick zu erweitern, der Gesundbleiben als eine aktive Leistung begreift und versucht, im Krankheitsgeschehen ein vorhandenes oder zu stimulierendes Gesundungspotential zu erkennen und für die Therapie fruchtbar zu machen. Denn in der Arzt-Patient-Beziehung stehen sich, wenn auch auf ganz unterschiedlichen Feldern, nämlich dem erlebten und gelebten Kranksein einerseits und den zu objektivierenden Krankheitsbefunden andererseits, durchaus zwei Experten gegenüber, die in einem wechselseitigen Perspektivenaustausch den diagnostisch-therapeutischen Prozess gestalten [6]. Insofern also der Patient nie nur ein biomedizinisch zugängliches Objekt ist, sondern stets auch ein geschichtliches Subjekt, dessen Erkrankungsund Gesundungsprozesse nicht nur dem Bios der Biologie unterliegen, sondern zugleich in den - ganz anders gearteten - 
Bios einer Biographie eingebunden sind, kann kaum ernsthaft das Erfordernis in Frage gestellt werden, einer zählenden eine erzählende Medizin an die Seite zu stellen bzw. die Schieflage einer einseitig biowissenschaftlich ausgerichteten Medizin durch ergänzende In-Dienst-Stellung hermeneutischer Erkenntnis- und Verstehensansätze auszutarieren. Für eine patienten- - und also nicht nur krankheitsorientierte - Praxis bedeutet dies nicht nur, patientenseitige Narrationen zuzulassen und ernst zu nehmen, sondern darüber hinaus die Ausbildung der Kompetenz, die am Patienten wahrgenommenen Zeichen und Symptome in einen erzählerischen Kontext einordnen und ihnen eine Bedeutung zuweisen zu können: 'The approach of the skilled diagnostician is the skill of an historian good at asking the right question and good at placing medical "fact" within the context of a temporal narrative that makes sense of the current constellation of symptoms and signs. A medical fact is a "medical" fact only within such a story' [7]. Erweisen sich Erkrankung und Gesundung aus naturwissenschaftlicher Perspektive als wertneutrale, apersonale und ungeschichtliche Prozesse, so zeigen sie sich aus lebensweltlicher Sicht als von Subjekten sinnhaft erfahrene biographische Einund Aufbrüche, denen im Spannungsfeld zwischen Herkunft und Zukunft des Kranken die Kategorie geschichtlicher Ereignishaftigkeit zukommt. Beide Aspekte, der biomedizinischnomothetische und der idiographisch-hermeneutische, lassen sich in der Praxis nicht getrennt voneinander verfolgen; sie sind unaufhebbar aufeinander bezogen. Im Zueinander-in-Beziehung-Setzen, im wechselseitigen Übersetzen des aus der einen Perspektive Gewonnenen in die andere besteht ja auch der ärztliche Auftrag und die spezifisch ärztliche Leistung. Insofern hat es auch keinen Sinn, den einen gegen den anderen Ansatz auszuspielen. Als Folge der Vorherrschaft biomedizinischen Denkens in der Medizin ist aber im Hinblick auf die Abfassung von Krankengeschichten und die Darstellung von Fallbeispielen eine «narrative Atrophie» [8] eingetreten, die ihrem Sujet, dem einen ganzen Kranken, keineswegs gerecht wird.

Daraus leitet sich freilich kein Plädoyer dafür ab, die im Rahmen der biomedizinischen Konzeption zu gewinnenden Beobachtungen und Schlussfolgerungen durch phantasiereiche Narrationen zu romantisieren. Gerade die Komplementärmedizin trägt noch immer an der Altlast, dass in der Vergangenheit eine Menge höchst unkritischer Kasuistiken publiziert wurde und gleichzeitig vielerorts die Meinung vertreten wurde, dass die verwandten Methoden wegen ihres individualisierenden Vorgehens einer Wirksamkeitsüberprüfung im Rahmen klinischer Studien nicht zugänglich seien. Vor dem Hintergrund einer zunehmenden Verbesserung der durch klinische Studien gestützten Beleglage zu Wirksamkeit und Nutzen komplementärmedizinischer Verfahren scheint mir aber die Zeit reif dafür, gute Kasuistik als ein unverzichtbares Instrument ärztlicher Erkenntnis- und Weiterbildung nicht nur wiederzuentdecken, sondern $\mathrm{zu}$ einem validen Werkzeug dafür weiterzuentwickeln, den einzelnen Fall in seiner Ver- flechtung von regelhaften und individuellen, austauschbaren und unaustauschbaren, biologischen und biographischen, ahistorischen und geschichtlichen Kategorien differenziert in den Blick zu bekommen. Damit möchte ich nicht einer Methodenverwässerung das Wort reden, wohl aber für einen Methodenpluralismus plädieren. Der naturwissenschaftlich-nomothetisch «versachlichte» Denk- und Kommunikationsstil besteht dort, wo er am Platz ist, ja durchaus zu Recht und sollte auch beim Abfassen von Fallberichten Orientierung gebend sein. Nur langt dies zu einer den Kranken als Person ins Auge fassenden ärztlichen Urteilsbildung nicht hin, woraus sich das in jüngster Zeit artikulierte Erfordernis ableitet, die Einseitigkeit der Evidence based Medicine durch die ergänzende Verfolgung und Ausarbeitung einer Narrative based Medicine zu überwinden [9]. Die besondere Qualität interessanter, lehrreicher und wirklichkeitsnaher Fallberichte bzw. Krankengeschichten zeigt sich, so ist meine Erfahrung, gerade in der Beherrschung und der Heranziehung beider Herangehensweisen und in der differenzierten Verknüpfung beider Aspekte. Die Verfolgung einer narrativen Kultur in der Medizin kann, indem sie den stets interpretativen und wertenden Charakter diagnostischer Aussagen hervorhebt, die grundsätzliche Kontext-, Standort- und damit Perspektivengebundenheit unseres Erkennens verdeutlichen, demzufolge Objektivierung gerade nicht Ausschluss, sondern methodische Einbeziehung der Subjektbezogenheit all unseres Wahrnehmens und Erkennens bedeutet.

Gerade die vertiefte Beschäftigung mit dem einzelnen Fall kann uns dazu erziehen, den Kranken nicht unter einer ex ante willkürlich festgelegten Sichtweise zu verfolgen, sondern nach der methodisch je angemessenen Form der Erkenntnisgewinnung zu suchen, also uns in der Einnahme unterschiedlicher Perspektiven und der Heranziehung verschiedenartiger Methoden zu erüben [5]. Dort, wo Einzelfallforschung sich die Aufgabe stellt, die reale Situation der ärztlichen Praxis auf ein höheres Reflexions- und Evidenzniveau zu heben, ist sie zugleich geeignet, die individuelle Erkenntnis- und Urteilsbildung des Arztes vor Ort zu verbessern - und damit die Einnahme einer investigativen Haltung nicht nur bei Studien-Patienten, sondern bei jedem Kranken zu fördern. Die Umsetzung expliziten Regelwissens macht dabei nur einen - und eher den weniger subtilen - Teil praktischen Handelns aus, der in nicht unerheblichem Umfang auf implizitem Wissen beruht. Die Praxis selbst hat ihre eigene wirklichkeitserschliessende Potenz, eine eigenständige Zugangsweise zu ihrem Gegenüber, was mit dem Phänomen einhergeht, dass sich unser Umgang mit und die Behandlung von Patienten mit ein und derselben (nosologischen) Diagnose beträchtlich - aber keineswegs nur auf subjektiver Willkür beruhend - unterscheiden kann.

Der konkrete Fall erweist sich so als der Dreh- und Angelpunkt, als Ort der Begegnung und der wechselseitigen Transformation von Theorie und Praxis, Allgemeinem und Besonderem, Regelhaftem und Individuellem, als Ausgangs- und 
Anwendungsort von propositionellem und implizitem Wissen, nomothetischer und idiographischer Forschung. Die Einsicht in die Irrtumsanfälligkeit der ärztlichen Urteilsbildung hat zum Gang in die grosse Zahl und zur Etablierung kontrollierter klinischer Studien und Metaanalysen auf dem Boden der schliessenden Statistik geführt. Dieser Ansatz ist aber seinerseits mit zahlreichen systemimmanenten Verzerrungen und Irrtumspotentialen behaftet, die der Praktiker kennen und beurteilen können muss, wenn ihm die vorgelegten Ergebnisse bei der Behandlung seiner Patienten als Grundlage für einen eigenständigen, individuellen Entscheidungsprozess und nicht als deren Ersatz und als Glaubensinhalte dienen sollen.

Mögen die an Kollektiven gewonnenen Daten noch so valide sein, sie entbinden den Arzt nicht von der Pflicht, über den Realitätswert und über die auf den konkreten Kranken bezogene Relevanz der ihm zur Verfügung gestellten externen Evidenz kraft seiner internen Evidenz ein Urteil zu fällen. Der Arzt muss sich bewusst sein, dass sein Handeln auch bei Anwendung von Massnahmen mit hohem evidenzbasierten Empfehlungsgrad noch eine Entscheidung unter Risiko ist. Sollen evidenzbasierte Leitlinien sich nicht zum Prokrustesbett der medizinischen Versorgung und soll sich die medizinische Versorgung nicht zu einem System der Eliminierung personaler Verantwortung auswachsen, dann stellt sich für den einzelnen Arzt die Aufgabe, das verfügbare wissenschaftliche Regelwissen zu instrumentalisieren, also im individuellen Fall auf seinen Wert hin beurteilen zu können. Für die Aneignung der hierzu notwendigen professionellen Kompetenz wird auch in Zukunft der gute Fallbericht eine ebenso zentrale wie unverzichtbare Rolle spielen.

\section{Literatur}

1 Walach H, Michalsen A: Praxis und Erkenntnis. Forsch Komplementärmed 2006; 13:4-5.

2 Wieland W: Diagnose. Überlegungen zur Medizintheorie. Bibliothek des skeptischen Denkens. Warendorf, Johannes G. Hoof, 2004.

3 Matthiessen PF: Die Therapieentscheidung des Arztes. Z Ärztl Fortbild Qual Gesundheitswes 2005;99:269-273.

4 Lorenz K: Über tierisches und menschliches Verhalten. Aus dem Werdegang der Verhaltenslehre. Gesammelte Abhandlungen, Bd 2. München, Pieper, 1965.

5 Antonovsky A: Salutogenese. Zur Entmystifizierung der Gesundheit. Dt erweiterte Herausgabe von A. Franke. Tübingen, dgvt, 1997.

6 Matthiessen PF: Der diagnostisch-therapeutische Prozess als Problem der Einzelfallforschung; in Ostermann T, Matthiessen PF (Hrsg): Einzelfallforschung in der Medizin. Bedeutung, Möglichkeiten und Grenzen. Frankfurt, VAS, 2003, pp 31-59.

7 Gatens-Robinson E: Clinical judgement and the rationality of human sciences. J Med Philos 1986;11: 167-178.

8 Marquard O: Über die Unvermeidlichkeit der Geisteswissenschaften; in Marquard O: Apologie des Zufälligen. Stuttgart., Reclam, 1986.

9 Greenhalgh T, Hurwitz B (Hrsg): Narrative-based Medicine, Sprechende Medizin. Bern, Huber, 2005. 